Decoding Nets and the Theory of Graphs

Author(s): Zdzislaw Pawlak

Source: Journal of the Society for Industrial and Applied Mathematics, Vol. 7, No. 1 (Mar., 1959), pp. 1-5

Published by: Society for Industrial and Applied Mathematics

Stable URL: http://www.jstor.org/stable/2099000

Accessed: 10/09/2008 02:39

Your use of the JSTOR archive indicates your acceptance of JSTOR's Terms and Conditions of Use, available at http://www.jstor.org/page/info/about/policies/terms.jsp. JSTOR's Terms and Conditions of Use provides, in part, that unless you have obtained prior permission, you may not download an entire issue of a journal or multiple copies of articles, and you may use content in the JSTOR archive only for your personal, non-commercial use.

Please contact the publisher regarding any further use of this work. Publisher contact information may be obtained at http://www.jstor.org/action/showPublisher?publisherCode=siam.

Each copy of any part of a JSTOR transmission must contain the same copyright notice that appears on the screen or printed page of such transmission.

JSTOR is a not-for-profit organization founded in 1995 to build trusted digital archives for scholarship. We work with the scholarly community to preserve their work and the materials they rely upon, and to build a common research platform that promotes the discovery and use of these resources. For more information about JSTOR, please contact support@jstor.org. 


\section{DECODING NETS AND THE THEORY OF GRAPHS*}

\section{ZI)ZISLAW PAWLAK}

Decoding nets are basic components of digital computers and other similar devices. The definition and the general theory of decoding nets are given in [3]. It is shown in this paper that the problem of minimizing decoding nets may be expressed in the language of the theory of graphs. (A decoding net may be assumed to be a graph; diodes are interpreted as branches, and wire connections between diodes as nodes of a graph). The necessary condition that a decoding net has the least number of diodes is given. In this paper, "decoding" has a little wider significance than in [3] and it will be defined exactly in what follows.

A graph $G$ is a set $N(G)$ of nodes together with a set $B(G)$ of branches (the two sets having no common elements) such that:

a. With each branch $A \in B(G)$, the ordered pair of nodes $(a, b) \in N(G)$ is associated. (We say that $A$ is directed from $a$ to $b$, and $a, b$ are called, respectively, the beginning and the end of the branch A.)

b. There is at most one branch between every pair of nodes $(a, b) \in N(G)$.

c. For all nodes $a \in N(G)$, there is a branch $A \in B(G)$ such that $a$ is the end or the beginning of $A$.

In this paper, we restrict ourselves to finite graphs, i.e., graphs for which $N(G)$ and $B(G)$ are both finite.

We denote by $\varphi_{N}(G)$ and $\varphi_{B}(G)$ the number of elements of $N(G)$ and $B(G)$ respectively. In the following, $\varphi(A)$ denotes the number of elements of the set $A$. A sequence $a_{0}, A_{1}, a_{1}, A_{2}, \cdots, A_{n}, a_{n},\left(n \geqq 1, a_{i} \in N(G)\right.$, $A_{i} \in B(G)$ ), will be called a way from $a_{0}$ to $a_{n}$ in $G$, if for all $i(1 \leqq i \leqq n)$, $a_{i-1}$ and $a_{i}$ are the beginning and the end of $A_{i}$ respectively.

If $(a, b) \in N(G)$, and there is exactly one way from $a$ to $b$ in $G$, we say that $a$ is connected to $b$ in $G$. (The case where there is more than one way from $a$ to $b$ in $G$ will not be considered in this paper.)

If $a$ is connected to $b$ in $G$, we will write $a$ Con $b$. Of course, the relation Con is not an equivalence relation.

A way which contains only two nodes is called simple.

We now introduce definitions of the relation Con between a set of nodes and a single node. These will be useful later. Let $M(G) \subset N(G)$ and $b \in N(G)-M(G)$. Then we define $M(G)$ Con $b$ to mean that $a \in M(G)$ implies $a$ Con $b$. Similarly, $b$ Con $M(G)$ means that $a \in M(G)$ implies $b$ Con $a$.

* Received by the editors February 13, 1957 and in revised form October 2, 1958. 
A graph $G$ is a tree if for all ordered pairs $(a, b) \in N(G)$ there is at most one way from $a$ to $b$ in $G$.

A graph $G$ is simple if all ways in it are simple. •

Corollary 1. Each simple graph is a tree.

If $a \in N(G)$, and there is no $b \in N(G)$ such that $a$ Con $b$, then $a$ is an output of $G$.

If $a \in N(G)$ and there is no $b \in N(G)$ such that $b$ Con $a$, then $a$ is an input of $G$.

Let $I(G)$ (or $O(G)$ ) be the set of all inputs (or outputs) of a graph $G$, partitioned into $n$ classes $K_{1}(G), K_{2}(G), \cdots, K_{n}(G)$ (i.e., $I(G)=\bigcup_{i=1}^{n} K_{i}(G)$ and $\bigcap_{i=1}^{n} K_{i}(G)=0$ ). The set $R_{I}(G)$ of $n$ elements $r_{1}, r_{2}, \cdots, r_{n}$ such that $r_{i} \in K_{i}(G)(i=1,2, \cdots, n)$ will be called a representation of a set $I(G)$. If $K_{1}, K_{2}, \cdots, K_{n}$ are nonempty classes, then the set $I(G)$ has $\prod_{i=1}^{n} \varphi\left(K_{i}(G)\right)$ different representations. For example, if $\varphi\left(K_{1}\right)=2$, $\varphi\left(K_{2}\right)=2, \varphi\left(K_{3}\right)=4, \varphi\left(K_{4}\right)=8$, then $I(G)$ has $2 \cdot 2 \cdot 4 \cdot 8(=126)$ different representations.

A graph $G$ is a decoding net (d.n.) if

(a) the set $O(G)$ of outputs is not empty,

(b) the set $I(G)$ can be partitioned into classes $K_{1}(G), K_{2}(G), \cdots$, $K_{n}(G), n \geqq 2$ such that $\varphi\left(K_{i}(G)\right) \geqq 2$, and such that

(c) for all representations $R_{I}(G)$ there exists at most one $a \in O(G)$ such that $R_{I}(G)$ Con $a$.

It is easy to prove the following.

Theorem 1. Let the directions of all branches in the d.n. $G$ be reversed. Call the resulting graph $G^{*}$. Then the set of outputs $O\left(G^{*}\right)$ can be partitioned into classes $K_{1}\left(G^{*}\right), K_{2}\left(G^{*}\right), \cdots, K_{n}\left(G^{*}\right)$, and for all representations $R_{o}(G)$, there exists at most one $a \in I\left(G^{*}\right)$ such that a Con $R_{o}\left(G^{*}\right)$.

Such a graph $G^{*}$ will be called a reverse decoding net, with respect to the d.n. $G$. In the following we will consider d.n.'s only; however all results concerning d.n.'s hold also for reverse decoding nets.

From the definition of a d.n. follows

Corollary 2. Each d.n. is a tree.

A d.n. is complete if for all $R_{I}(G)$ there exists exactly one $a \in O(G)$ such that $R_{I}(G)$ Con $a$ and for all $a \in O(G)$ there exists exactly one $R_{I}(G)$ such that $R_{I}(G)$ Con $a$. A complete d.n. is abbreviated c.d.n.

THEOREM 2. For an arbitrary c.d.n. $G$,

$$
\varphi(O(G))=\prod_{i=1}^{n} \varphi\left(K_{i}(G)\right)
$$

Let $G$ and $H$ be c.d.n.'s. Then the d.n. $G \cup H$ formed so that $O(G)=$ 
$K_{i}(H)$ will be called a superposition of the d.n. $G$ into a d.n. $H$ and will be denoted by Sup $K_{i}(H) / G$. We remark that a superposition of $G$ into $H$ does not always exist.

From the definition of a superposition, we have

Corollary 3. If $G$ and $H$ are c.d.n.'s, then Sup $K_{i}(H) / G$ is also a c.d.n.

We now give a recursive definition of decomposable c.d.n.'s.

a. Simple c.d.n.'s are decomposable (into themselves).

b. If $G$ and $H$ are decomposable c.d.n.'s, then the c.d.n.'s Sup $K_{i}(H) / G$ are also decomposable (if $\operatorname{Sup} K_{i}(H) / G$ exists).

In the remainder of this paper, by c.d.n. we will understand a decomposable c.d.n. only.

Let $A_{n}(n \geqq 2)$ denote a sequence of $K$ integers $a_{i}$ separated by "periods" such that $a_{i} \geqq 2, i=1,2, \cdots, n$. $A_{n}$ is a formula if it contains parentheses "(", ")". For example 2.8.(5.)3) is a formula. A formula is wellformed if it contains properly paired $\dagger$ parentheses. The recursive definition of well-formed formulas (w.f.f.'s) is given below.

a. $\left(A_{n}\right)$ is a w.f.f. (for example-(2.2), (2.2.2), (3.8.4)).

b. If $\alpha$ and $\beta$ are w.f.f.'s, then replacing $a_{i}$ in $\beta$ by $\alpha$ or in symbols

$\operatorname{Sup}\left(\begin{array}{c}\beta \\ a_{i}\end{array}\right) \alpha$ is also a w.f.f. For example, we have

$$
\operatorname{Sup}\left(\begin{array}{c}
(2.4) \\
4
\end{array}\right)(2.2)=(2 .(2.2))
$$

A w.f.f. with $k$ pairs of parentheses will be denoted by $A_{n}{ }^{k}$. In particular, $A_{n}{ }^{\prime}=\left(A_{n}{ }^{0}\right)$.

From the definitions of a decomposable c.d.n. and a w.f.f. we have

LEmma 1. The set of all decomposable c.d.n.'s and the set of all w.f.f.'s are isomorphic.

Thus w.f.f.'s may be assumed to be the names of c.d.n.'s. The w.f.f.'s are associated with c.d.n.'s in the following way.

a. If $G$ is a simple c.d.n., with inputs partitioned into classes $K_{1}(G)$, $K_{2}(G), \cdots, K_{n}(G)$, then the w.f.f. associated with it has the form

$$
\left(\varphi\left(K_{1}(G)\right) . \varphi\left(K_{2}(G)\right) . \cdots . \varphi\left(K_{n}(G)\right)\right) .
$$

b Let $F(G)$ and $F(H)$ denote w.f.f.'s associated with c.d.n.'s $G$ and $H$ respectively. With the e.d.n. $\operatorname{Sup} K_{i}(H) / G$ we associate the w.f.f. $\operatorname{Sup}\left(\begin{array}{c}F(H) \\ a_{i}\end{array}\right) F(G)$ where $a_{2}=\varphi\left(K_{i}(G)\right)$. c.d.n.'s $A$ and $B$ are equivalent if and only if the w.f.f.'s $A$ and $B$ differ at most in (1) the order of factors,

† Parentheses occurring in a formula are said to be properly paired if there are as many left-hand parentheses as there are right-hand parentheses. 
(2) the number of parentheses, and

(3) the location of parentheses.

Thus each set of integers $a_{1}, a_{2}, \cdots, a_{n}, n \geqq 2, a_{i} \geqq 2$ defines a class of equivalent c.d.n.'s.

From the definition of a simple c.d.n. we have

Lemma 2. $\varphi_{B}\left(A_{n}{ }^{1}\right)=n \prod_{i=1}^{n} a_{i}$.

LemMA 3. $\varphi_{N}\left(A_{n}{ }^{1}\right)=\prod_{i=1}^{n} a_{i}+\sum_{i=1}^{n} a_{i}$.

Lemma 4 . A c.d.n. $A_{n}{ }^{k}$ is decomposable into kimple c.d.n.'s. From Lemmas 2 and 4 follows

THEOREM 3. $\varphi_{B}\left(A_{n}{ }^{k}\right)=\sum_{i=1}^{n} p_{r} \prod_{r} a_{i}$ where $\prod_{r} a_{i}$ denotes the product of factors joined by the rth pair of parentheses, and $p_{r}$ the number of factors in the rth pair of parentheses.

We assume that each pair of parentheses in the w.f.f. $A_{n}{ }^{k}$ is numbered with the numbers $1,2, \cdots, k$; however the manner of numeration is in our case not important. From Lemmas 3 and 4 we have

THEOREM 4. $\varphi_{N}\left(A_{n}{ }^{k}\right)=\sum_{i=1}^{k} \prod_{r} a_{i}+\sum_{i=1}^{n} a_{i}$.

Theorem 5. A necessary and sufficient condition for $\varphi_{N}\left(A_{n}{ }^{k}\right)$ to be minimal for a given $a_{1}, \cdots, a_{n}$ is that $k=1$.

THEOREM 6. A necessary condition for $\varphi_{B}\left(A_{n}{ }^{k}\right)$ to be minimal for a given $a_{1}, \cdots, a_{n}$ is that $k=n-1$, i.e., each pair of parentheses encloses two factors.

Proof. This theorem is equivalent to the inequality

$$
\varphi_{B}\left(A_{n}{ }^{k}\right) \geqq \varphi_{B}\left(A_{n}^{k+1}\right)
$$

for $k=1,2, \cdots, n-2$. Let $A_{n}{ }^{k}$ have the form

$$
\left(x_{1}, x_{2}, \cdots, x_{t}\right)
$$$$
(t<n),
$$

where each $x_{i}$ is either a single term $a_{i}$ or a product with $u_{i}$ terms. We may assume that each $x_{i}$ has $u_{i}-1$ parentheses (and the whole formula has $k$ parentheses). Thus from (1), by Lemma 2 and Theorem 3 , we obtain

$$
\sum_{i=1}^{t} \varphi_{B}\left(x_{i}\right)+t \prod_{i=1}^{t} x_{i} \geqq \sum_{i=1}^{t} \varphi_{B}\left(x_{i}\right)+(t-p+1) \prod_{i=1}^{t} x_{i}+p \prod_{k+1} x_{i},
$$

where $p$ is the number of factors contained in the $(k+1)$ th pair of parentheses.

From (3), we have

$$
\rho \leqq 1-\frac{1}{p}
$$


where $\rho=1 / \prod \overline{k+1} x_{i}$ and $\prod \overline{k+1} x_{i}$ denotes the product of all factors in (2), with the exception of those contained in the $(k+1)$ th pair of parentheses. Because $\prod_{\overline{k+1}} x_{i} \geqq 2$ and $p \geqq 2$, the theorem is proved.

The condition is not necessary when $\prod_{\overline{k+1}} x_{i}=2$ and $p=2$; for example, if $A_{3}{ }^{1}=(2.2 .2)$, and $A_{3}{ }^{2}=(2 .(2.2))$, then $\varphi_{B}\left(A_{3}{ }^{1}\right)=3 \cdot 8=24, \varphi_{B}\left(A_{3}{ }^{2}\right)=$ $2 \cdot 4+2 \cdot 8=24 ;$ or if $B_{3}{ }^{1}=(2.3 .4), B_{3}{ }^{2}=(2 .(3.4))$, then $\varphi_{B}\left(B_{3}{ }^{1}\right)=$ $3 \cdot 2 \cdot 3 \cdot 4=72, \varphi_{B}\left(B_{3}{ }^{2}\right)=2 \cdot 3 \cdot 4+2 \cdot 2 \cdot 3 \cdot 4=72$. But we can say that the minimum case can always be attained with $k-1$ parentheses.

It would be interesting to give a necessary condition that $\varphi_{B}\left(A_{n}^{n-1}\right)$ be minimal. However, in the general case it seems to be rather difficult.

I am indebted to Professor W. T. Tutte of the University of Toronto and Mr. A. Wakulicz of the Institute of Mathematics in Warsaw for their helpful suggestions.

\section{REFERENCES}

1. I). R. BRoWN AND N. ROchester, Rectifier networks for multiposition switching, Proc. Inst. Radio Engrs., 37 (1949), pp. 139-147.

2. The Annals of the Computation Laboratory of Harvard University: Synthesis of electronic computing and control circuits by the staff of the computation laboratory, Harvard Univ. Press, Cambridge, 1951, vol. 27, pp. 126-144.

3. Arthur W. Burks, Robert MCNaughton, Carl H. Pollmar, Don W. Warren AND JESSE B. WRIGHT, Complete decoding nets: general theory and minimality, J. Soc. Indust. Appl. Math., 2(1954), pp. 201-243.

Warsaw Polytechnic Institute

Warsaw, Poland 